\title{
Prognostic Significance of p27 and Ki-67 Expression in Mucoepidermoid Carcinoma of the Intraoral Minor Salivary Gland
}

\author{
Mitsukuni Okabe, D.M.D., Hiroshi Inagaki, M.D., Takayuki Murase, M.D., Masahisa Inoue, D.M.D., \\ Noriyuki Nagai, D.M.D., Tadaaki Eimoto, M.D. \\ Department of Pathology (MO, HI, TM, TE), Nagoya City University Medical School, Nagoya, Japan; and \\ Department of Oral Pathology (MI, NN), Okayama University Dental School, Okayama, Japan
}

p27 and Ki-67, a universal cyclin-dependent kinase inhibitor and a proliferative cell marker, respectively, have been useful in predicting clinical aggressiveness in various human tumors. We studied clinicopathologic significance of these molecules in mucoepidermoid carcinoma of the intraoral minor salivary gland. Expression of p27 and Ki-67 was assessed immunohistochemically in primary mucoepidermoid carcinomas from 31 patients without distant metastasis at surgery. Correlation each of p27 and Ki-67 expression was analyzed with various clinicopathologic parameters including age, sex, primary tumor site, tumor size, nodal metastasis, clinical stage, and histologic grade. The latter was evaluated using a point-scoring scheme of Auclair et $a l$. that consists of five histologic factors (intracystic component, neural invasion, necrosis, mitosis, and anaplasia). p27 expression was correlated inversely with histologic grade $(P=.007)$, but with none of other factors. When the correlation of p27 expression was further examined with each of the histologic factors, it was correlated significantly with intracystic component, but not with neural invasion, necrosis, mitosis, or anaplasia. Ki-67 expression was correlated significantly with histologic grade only in the clinicopathologic factors $(P<.0001)$, and in the histologic factors, with necrosis, mitosis, and anaplasia. Multivariate prognostic analyses were performed to identify independent risk factors for both disease-free and overall survivals. Large tumor size $(P=.031$, relative risk $=5.5)$ and low p27 expression $(P=.012$, relative risk $=5.2)$ were risk factors for worse disease-free survival. Low p27 ex-

Copyright (C) 2001 by The United States and Canadian Academy of Pathology, Inc.

VOL. 14, NO. 10, P. 1008, 2001 Printed in the U.S.A.

Date of acceptance: May 25, 2001.

Address reprint requests to: Hiroshi Inagaki, M.D., Department of Pathology, Nagoya City University Medical School, 1 Kawasumi, Mizuho-ku, Nagoya 467-8601, Japan; e-mail: hinagaki@med.nagoya-cu.ac.jp; fax: 81-52-851-4166. pression $(P=.015$, relative risk $=15.2)$ was selected as a risk factor for worse overall survival. Other factors including age, sex, tumor site, nodal status, clinical stage, histologic grade, and Ki-67 did not emerge as independent risk factors in either prognostic analysis. These data suggest that $\mathrm{p} 27$ may be useful in estimating prognosis of the patients who have mucoepidermoid carcinoma of the intraoral minor salivary gland.

KEY WORDS: p27, Ki-67, Histologic grade, Intraoral mucoepidermoid carcinoma, Prognosis, Survival analysis.

Mod Pathol 2001;14(10):1008-1014

Mucoepidermoid carcinoma (MECA) is the most common malignant tumor of the minor salivary gland in the oral cavity (1). However, most of the previous clinicopathologic studies for MECA include cases of both major and minor salivary glands. The number of such studies restricted to MECA of the intraoral minor salivary gland is limited (2-6), and prognostic factors for this tumor have been poorly clarified.

Recently, some cell cycle-associated molecules have been useful in predicting survival of the patients with various malignant tumors. p27 is a member of the universal cyclin-dependent kinase inhibitor family (7). p27 blocks the cell cycle at the G1/S phase checkpoint and is highly expressed in cells arrested at the G0 and G1 phases. Enhanced p27 expression is induced by cell-cell contact and specific growth factors, such as transforming growth factor- $\beta(8,9)$ and cyclic AMP (10). Prognostic value of p27 expression has been reported in various human tumors (11). The low p27 expression was associated with an unfavorable prognosis for squamous cell carcinoma of the tongue (12). However, the significance of $\mathrm{p} 27$ has not been studied in MECA of either major or minor salivary gland origin. 
The Ki-67 antibody recognizes proliferating cells, but not resting cells (13). Clinical significance of Ki-67 expression as a proliferative cell and a prognostic marker has been investigated in many types of human tumors including those of salivary gland origin such as acinic cell and adenoid cystic carcinomas $(14,15)$. The prognostic value of Ki-67 expression was reported in MECA cases including both major and minor salivary gland origin (16) and recently in MECA cases restricted to the minor salivary gland origin (6). However, importance of Ki-67 as an independent risk factor by means of multivariate prognostic analysis was not verified in these reports.

In the present study, we investigated expression of p27 and Ki-67 by immunohistochemistry in 31 cases of MECAs of intraoral minor salivary gland. Prognostic impact of p27 and Ki-67 was evaluated using multivariate analyses for disease-free and overall survivals. Low p27 expression emerged as an independent risk factor for both survivals, suggesting that this molecule may be a useful marker for estimating clinical aggressiveness of MECA of the minor salivary gland in the oral cavity.

\section{MATERIALS AND METHODS}

\section{Tumor Cases}

MECAs of the intraoral minor salivary gland were retrieved from the files of the Department of Pathology, Nagoya City University Medical School and the Department of Oral Pathology, Okayama University Dental School between 1977 and 1999. All tumors were re-evaluated, using hematoxylin and eosin and mucicarmine stained sections. Only patients without distant (other than the regional lymph node) metastasis at surgery were included in this study. All tumors were surgically treated with curative intent. There were 31 tumors surgically resected from 31 patients. The tumors had been fixed in $10 \%$ buffered formalin and embedded in paraffin.

\section{Clinicopathologic Data}

The clinical factors retrieved were as follows: age, sex, primary tumor site, tumor size, metastasis to regional lymph nodes at surgery, clinical stage, histologic grade, and follow-up status. The clinical stage was determined according to the criteria used for oral squamous cell carcinoma as recommended by Spiro et al. (17). The tumors were histologically graded according to a grading system advocated for intraoral MECA by Auclair et al. (5). The grades (low, intermediate, and high) were determined by the sum of point values assigned to each of five histologic factors (intracystic component, neural invasion, necrosis, mitosis, and anaplasia). Patient outcome was verified and updated through the medical records. The day of the primary surgery was considered as the start of the follow-up. Five patients received pre- or postoperative chemotherapy, and five patients postoperative radiation therapy.

\section{Immunohistochemistry}

Primary monoclonal antibodies used were as follows; anti-p27 (K25020, Transduction Laboratory, Lexington, $\mathrm{NY}$, reacted at the concentration of 0.5 $\mu \mathrm{g} / \mathrm{mL}$ ) and anti-Ki-67 antigen (MIB-1, Immunotech S.A., Marseilles, France, concentration of 4 $\mu / \mathrm{mL}$ ). Immunostaining was performed with the streptavidin-biotin method (Histofine SAB kit, Nichirei, Tokyo, Japan) as previously described (18). Paraffin sections cut at $3 \mu \mathrm{m}$ were deparaffinized and heat-treated with $10 \mathrm{~mm}$ citrate buffer, pH 6.0, in a pressure cooker for 15 minutes for antigen retrieval. After developing the immunosignals, sections were counterstained with hematoxylin. Positive controls for the p27 and Ki-67 immunostaining consisted of tonsillar tissues; p27 was positive for small lymphocytes in the mantle zones, and Ki-67 was positive for proliferating lymphocytes in the germinal centers. Negative controls consisted of substituting normal mouse serum for the primary antibodies and reactions without primary or secondary antibody.

\section{Scoring Technique}

Scoring and interpretation of immunohistochemical results were performed by two of the authors (MO and TM) without any knowledge of clinical outcome and other clinical data. The areas showing the representative p27 or Ki-67 expression in each section were chosen, and the minimum of 1,000 tumor cells were counted under light microscopic fields $(\times 400)$ using a computer-assisted image-analyzing system (VM-30; Olympus, Tokyo, Japan). The percentage of positive tumor cell nuclei was recorded as labeling index (LI). Cut-off values of $15 \%$ and $10 \%$ for p27 and Ki-67, respectively, showed superior segregation into prognostic groups, and the cases were divided into two groups, high and low expressors.

\section{Statistical Analysis}

Correlation each of p27 and Ki-67 expression with various clinicopathologic parameters was analyzed by the Fischer's exact test. Association between p27 and Ki-67 was estimated by unpaired $t$ test and simple regression analysis. To identify the parameters significantly associated with diseasefree and overall survivals of the patients, survival 
rate was calculated by Kaplan-Meier method, and the statistical difference was analyzed by log-rank test. The multivariate analysis was performed using the Cox's proportional hazard models; the parameters significant in the log-rank univariate test were included in the stepwise regression procedure, which was discontinued when the $P$ value for a new variable entering was greater than .05 . All of the analyses were performed using statistical package StatView v.4.5.1 (Abacus Concepts, Berkeley, CA).

\section{RESULTS}

\section{Patients' Profile}

Thirty-one patients with primary MECA of the intraoral minor salivary gland were subjected to the present study. Seventeen of 31 cases $(55 \%)$ were more than 60 years old at surgery (range from 13 to 83 years, median 62 years). There was a mild female predilection with a male:female ratio of 1:1.4. The site of primary tumor was the palate in seven cases, tongue in seven, oral floor in six, retromolar area in six, mandible in two, and other sites in three (each in the tonsillar area, lip, and buccal mucosa). Seventeen tumors were more than $2 \mathrm{~cm}$ in diameter. Eight cases showed metastasis to the regional cervical lymph nodes at surgery. Fourteen patients were at stage I, three patients at stage II, 12 patients at stage III, and two patients at stage IV. Follow-up period ranged from 6 to 263 months with a mean of 55 months. Twenty-five patients were alive with no evidence of disease; five died of the disease; and one died of other causes.

\section{Tumor Grade and Expression of p27 and Ki-67}

According to the criteria by Auclair et al. (5), the tumors were divided into 19 low-, 3 intermediate-, and 9 high-grade cases. Immunohistochemistry for p27 showed a distinct nuclear staining (Fig. 1, A-B). p27 LI ranged from 0.5 to $94.8 \%$ with a median of $30.6 \%$, and Ki-67 LI from 0.4 to $89.1 \%$ with a median of $6.1 \%$. There were 24 high p27 expressors and 11 high Ki-67 expressors. Correlation each of p27 and Ki-67 expression with various clinicopathologic factors is shown in Table 1. p27 expression was inversely correlated with histologic grade $(P=.007)$, but with none of other factors (age, sex, tumor site, tumor size, nodal metastasis, and clinical stage). Because the histologic grade consists of five histologic factors (intracystic component, neural invasion, necrosis, mitosis, and anaplasia), the correlation of p27 expression with each of these histologic factors was further examined (Table 1). The intracystic component was the only factor that correlated with p27 expression. Similarly, Ki-67 expression correlated with only histologic grade $(P<$
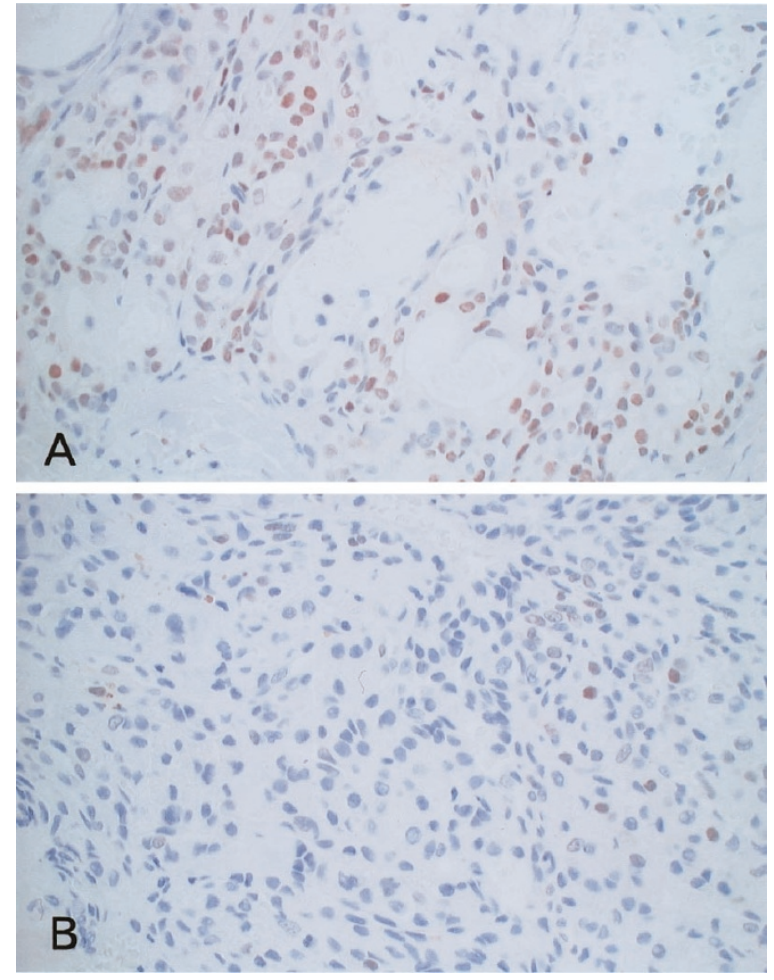

FIGURE 1. Representative expression of p27 in high- and low-grade mucoepidermoid carcinomas. p27 is highly expressed in a low-grade mucoepidermoid carcinoma (MECA) case (A), whereas its expression is low in a high-grade MECA case $(\mathbf{B})$. Original magnification $\times 400$, A and B.

.0001) among various clinicopathologic factors. Histologic factors that showed correlation with Ki-67 expression were necrosis, mitosis, and anaplasia.

\section{Correlation Between p27 LI and Ki-67 LI}

The difference in Ki-67 LI between the high and low p27 expressors was evaluated. The Ki-67 LI in the high p27 LI group $(13.3 \pm 4.6$, mean \pm standard error) was somewhat lower than that in the low p27 LI group $(25.3 \pm 6.3)$, but the difference was not statistically significant. The linear regression analysis showed that there was little association between p27 LI and Ki-67 LI $\left(r^{2}=.005\right)$.

\section{Survival Analyses}

The disease-free 5-year survival rate was 55\%, and the overall 5 -year survival rate was $80 \%$. The following clinicopathologic factors were included in the survival analyses: age, sex, tumor site, tumor size, nodal metastasis, clinical stage, histologic grade, p27 expression, and Ki-67 expression. The disease-free and overall 5-year survival rates stratified by these factors are shown in Table 2. To identify the factors useful in predicting disease-free and overall survivals, uni- and multivariate analyses were performed (Table 3). For the disease-free sur- 
TABLE 1. Correlation of p27 and Ki-67 Expression with Various Clinicopathologic Factors (n = 31 )

\begin{tabular}{|c|c|c|c|c|c|c|c|}
\hline \multirow{2}{*}{ Variables } & & \multicolumn{3}{|c|}{ p27 LI } & \multicolumn{3}{|c|}{ Ki-67 LI } \\
\hline & & Low & High & $P$ & Low & High & $P$ \\
\hline \multirow[t]{2}{*}{ Age } & $\leq 60$ & 3 & 11 & & 11 & 3 & \\
\hline & $>60$ & 4 & 13 & N.S. & 9 & 8 & N.S. \\
\hline \multirow[t]{2}{*}{ Sex } & Male & 3 & 10 & & 6 & 7 & \\
\hline & Female & 4 & 14 & N.S. & 14 & 4 & N.S. \\
\hline \multirow[t]{2}{*}{ Tumor site } & Tongue and oral floor & 3 & 10 & & 6 & 7 & \\
\hline & Other sites ${ }^{b}$ & 4 & 14 & N.S. & 14 & 4 & N.S. \\
\hline \multirow[t]{2}{*}{ Tumor size } & $\leq 2 \mathrm{~cm}$ & 2 & 12 & & 11 & 3 & \\
\hline & $>2 \mathrm{~cm}$ & 5 & 12 & N.S. & 9 & 8 & N.S. \\
\hline \multirow[t]{2}{*}{ Nodal status $^{a}$} & Negative & 4 & 19 & & 17 & 6 & \\
\hline & Positive & 3 & 5 & N.S. & 3 & 5 & N.S. \\
\hline \multirow[t]{2}{*}{ Clinical stage } & I, II & 2 & 15 & & 13 & 4 & \\
\hline & III, IV & 5 & 9 & N.S. & 7 & 7 & N.S. \\
\hline \multirow[t]{2}{*}{ Histologic grade } & Low & 1 & 18 & & 18 & 1 & \\
\hline & Intermediate + high & 6 & 6 & 0.007 & 2 & 10 & $<.0001$ \\
\hline \multirow[t]{2}{*}{ Intracystic component } & $\geq 20 \%$ & 0 & 14 & & 11 & 3 & \\
\hline & $<20 \%$ & 7 & 10 & 0.009 & 9 & 8 & N.S. \\
\hline \multirow[t]{2}{*}{ Neural invasion } & Negative & 6 & 24 & & 20 & 10 & \\
\hline & Positive & 1 & 0 & N.S. & 0 & 1 & N.S. \\
\hline \multirow[t]{2}{*}{ Necrosis } & Negative & 5 & 23 & & 20 & 8 & \\
\hline & Positive & 2 & 1 & N.S. & 0 & 3 & .037 \\
\hline \multirow[t]{2}{*}{ Mitotic figure } & $<3 / 10$ HPFs & 2 & 17 & & 18 & 1 & \\
\hline & $\geq 4 / 10 \mathrm{HPFs}$ & 5 & 7 & N.S. & 2 & 10 & $<.0001$ \\
\hline \multirow[t]{2}{*}{ Anaplasia } & Negative & 3 & 19 & & 19 & 3 & \\
\hline & Positive & 4 & 5 & N.S. & 1 & 8 & .0002 \\
\hline
\end{tabular}

${ }^{a}$ Regional lymph node metastasis.

${ }^{b}$ Palate, retromolar area, mandible, tonsillar area, lip, and buccal mucosa.

LI, labeling index; N.S., not significant.

TABLE 2. Five-Year Survival Rates for Various Clinicopathologic Factors, p27 LI, and Ki-67 LI

\begin{tabular}{|c|c|c|c|}
\hline Variables & & $\begin{array}{c}\text { Disease- } \\
\text { Free } \\
\text { Survival (\%) }\end{array}$ & $\begin{array}{c}\text { Overall } \\
\text { Survival (\%) }\end{array}$ \\
\hline All patients & & 55 & 80 \\
\hline \multirow[t]{2}{*}{ Tumor site } & Tongue and oral floor & 35 & 83 \\
\hline & Other sites ${ }^{b}$ & 69 & 77 \\
\hline \multirow[t]{2}{*}{ Tumor size } & $\leq 2 \mathrm{~cm}$ & 75 & 100 \\
\hline & $>2 \mathrm{~cm}$ & 38 & 65 \\
\hline \multirow[t]{2}{*}{ Nodal status $^{a}$} & Negative & 67 & 88 \\
\hline & Positive & 19 & 54 \\
\hline \multirow[t]{2}{*}{ Clinical stage } & I, II & 73 & 100 \\
\hline & III, IV & 33 & 57 \\
\hline \multirow[t]{2}{*}{ Histologic grade } & Low & 60 & 95 \\
\hline & Intermediate + high & 50 & 54 \\
\hline \multirow[t]{2}{*}{ p27 LI } & Low & 19 & 33 \\
\hline & High & 65 & 96 \\
\hline \multirow[t]{2}{*}{ Ki-67 LI } & Low & 61 & 95 \\
\hline & High & 44 & 53 \\
\hline
\end{tabular}

${ }^{a}$ Regional lymph node metastasis.

${ }^{b}$ Palate, retromolar area, mandible, tonsillar area, lip, and buccal mucosa.

LI, labeling index.

vival, large tumor size $(P=.013)$, regional lymph node metastasis $(P=.0054)$, advanced clinical stage $(P=.0089)$, and low p27 expression $(P=.0032)$ were selected as risk factors in the univariate analysis. When the stepwise multivariate analysis was performed on these four factors, large tumor size ( $P$ $=.031$, relative risk $=5.5$ ) and low p27 expression $(P=.012$, relative risk $=5.2)$ remained as independent risk factors. For the overall survival, large tumor size $(P=.0037)$, nodal metastasis $(P=.034)$, advanced clinical stage $(P=.0091)$, high histologic grade $(P=.034)$, low p27 expression $(P=.0011)$, and high Ki-67 expression $(P=.024)$ emerged as significant risk factors in the univariate analysis. In the stepwise multivariate analysis entering these six factors, p27 LI was the only factor that achieved the statistical significance $(P=.015$, relative risk $=$ 15.2). Disease-free survivals analyzed for tumor size and p27 expression and overall survival for p27 expression are shown in Figure 2.

\section{DISCUSSION}

The clinical features of our MECA cases of the intraoral minor salivary gland were in accord with those of the previous reports: a peak incidence in the middle age (range, 40 to 70 years), mild female predilection, and the palate being the most common location for the tumor (2-6). In contrast to these basic statistics, the survival data and prognostic factors have been poorly analyzed for this carcinoma; tumor size (4) and proliferation- and apoptosis-related factors (6) were previously implicated as risk factors for overall survival by univariate analysis. In the present study, we showed the 5 -year survival rates stratified for various factors (Table 2). Furthermore, we analyzed independent risk factors for disease-free and overall survivals using Cox's multivariate regression model. When age, sex, tumor size, nodal metastasis, tumor site, clinical stage, histologic grade, p27 expression, and Ki-67 expression were included as parameters for the survival analyses, p27 was an independent fac- 


\begin{tabular}{|c|c|c|c|c|c|}
\hline \multirow{2}{*}{ Variables } & & \multicolumn{2}{|c|}{ Disease-Free Survival $(P)$} & \multicolumn{2}{|c|}{ Overall Survival $(P)$} \\
\hline & & Univariate & Multivariate & Univariate & Multivariate \\
\hline \multirow[t]{2}{*}{ Age } & $\leq 60$ & & & & \\
\hline & $>60$ & N.S. & & N.S. & \\
\hline \multirow[t]{2}{*}{ Sex } & Male & & & & \\
\hline & Female & N.S. & & N.S. & \\
\hline \multirow[t]{2}{*}{ Tumor site } & Tongue and oral floor & & & & \\
\hline & Other sites ${ }^{b}$ & N.S. & & N.S. & \\
\hline \multirow[t]{2}{*}{ Tumor size } & $\leq 2 \mathrm{~cm}$ & & & & \\
\hline & $>2 \mathrm{~cm}$ & . 013 & .031 & .037 & N.S. \\
\hline \multirow[t]{2}{*}{ Nodal status $^{a}$} & Negative & & & & \\
\hline & Positive & .0054 & N.S. & .034 & N.S. \\
\hline \multirow[t]{2}{*}{ Clinical stage } & I, II & & & & \\
\hline & III, IV & .0089 & N.S. & .0091 & N.S. \\
\hline \multirow{2}{*}{ Histologic grade } & Low & & & & \\
\hline & Intermediate + high & N.S. & & .034 & N.S. \\
\hline \multirow[t]{2}{*}{ p27 LI } & Low & & & & \\
\hline & High & .0032 & .012 & .0011 & .015 \\
\hline \multirow[t]{2}{*}{ Ki-67 LI } & Low & & & & \\
\hline & High & N.S. & & .024 & N.S. \\
\hline
\end{tabular}

${ }^{a}$ Regional lymph node metastasis.

${ }^{b}$ Palate, retromolar area, mandible, tonsillar area, lip, and buccal mucosa.

LI, labeling index; N.S., not significant.

tor in estimating both disease-free and overall survivals of the patients. Tumor size was another independent risk factor for disease-free survival (Table 3). Ki-67, which may be a risk factor for MECA of the salivary gland $(6,16)$, was selected as a risk factor in the univariate analysis for overall survival. However, it did not achieve a statistical significance in the multivariate analysis. MECAs of the tongue and oral floor were previously suggested to be more aggressive than those of other sites in the oral cavity (5), nevertheless, the primary tumor site (tongue and oral floor versus others) was not a significant factor for either disease-free or overall survival in this study (Table 3 ).

Auclair et al. (5) advocated a histologic grading system for MECA of the intraoral minor salivary glands where the grades (low, intermediate, and high) are determined by the sum of point values assigned to each of five histologic factors (intracystic component, neural invasion, necrosis, mitosis, and anaplasia). The prognostic usefulness of this histologic grading system has not been verified after it appeared in the literature. We classified our cases using this grading system and showed its prognostic value in the univariate analysis for overall survival. Although it did not emerge as a prognostic factor in the multivariate analysis, the grading system is useful for selecting the high risk cases in the routine histologic diagnosis because the prognosis could be estimated simply by a hematoxylin and eosin stained section. The clinical behavior of intermediate grade tumor has not been fully determined (5). In our study, when considering the intermediate grade as potentially high grade (high plus intermediate versus low grade), a significant difference in overall survival was obtained (Table
3). However, other combinations (high versus intermediate versus low; high versus intermediate plus low) did not give the statistical significance (data not shown). This finding suggests that intermediate grade cases may behave as aggressively as highgrade cases.

p27 regulates progression from the G1 to S phase of the cell cycle by inhibiting cyclin-D or -E dependent kinase activity (9). Mutation and/or functional inactivation of the p27 gene has not been reported in human tumors, and the reduction in the amount of p27 protein appears to be due to increased p27 degradation through a proteasome pathway (19). We recently showed that suppression of p27 mRNA also plays a role in the reduction of p27 protein using human tumor cell lines (20). Several extracellular signals, including those of transforming growth factor- $\beta$ (9) and cyclic AMP (10), and cellcell contact $(8,20)$ are also important in upregulation of p27 expression. In this study, p27 expression was correlated with histologic grade (Table 1), especially intracystic component, i.e., space occupied by cyst. This factor is considered to represent tumor cellularity or solid growth of the tumor. Seven of 19 cases exhibiting higher cellularity (intracystic component less than 20\%) showed low p27 expression (Table 1), and five of these seven cases showed poor clinical course (four patients died of the tumor and one showed tumor recurrence). It is intriguing to speculate that, in these tumors, the mechanism of p27 up-regulation through cell-cell contact may be impaired. This finding should be further studied to elucidate the precise role of p27 in intraoral MECAs.

The prognostic significance of Ki-67 has also been reported in various tumors. Ki-67 was re- 
A

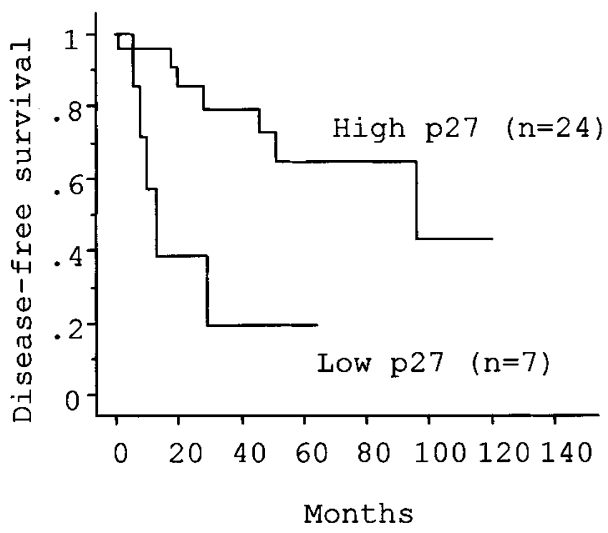

B

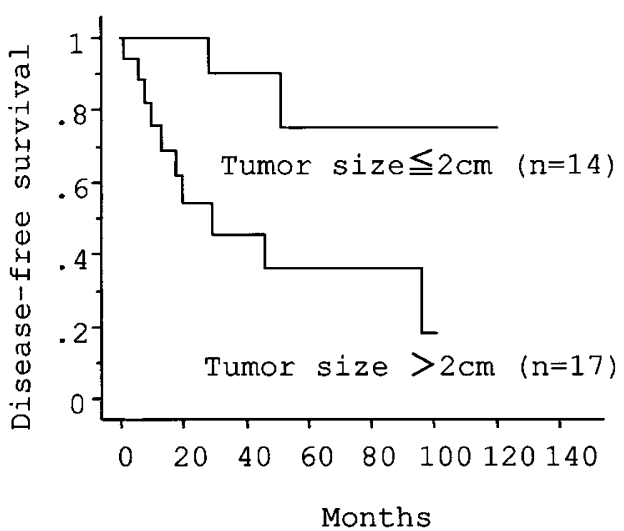

C

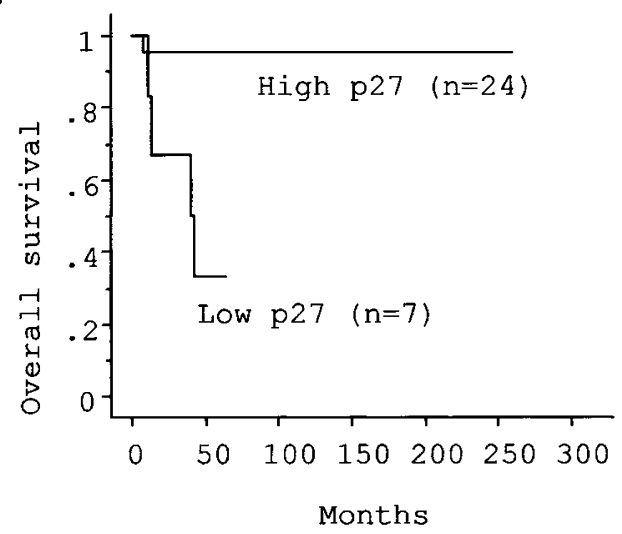

FIGURE 2. Kaplan-Meier plots for disease-free and overall survivals of patients with intraoral minor salivary gland mucoepidermoid carcinoma. A, Disease-free survival for p27 expression. B, Disease-free survival for tumor size. C, Overall survival for p27 expression.

ported as a prognostic factor in a study including both major and minor salivary gland MECAs (16) and in a study including the minor salivary gland MECAs (6). In the present analysis restricted to MECAs of the minor salivary gland origin, Ki-67 LI was also selected as a risk factor for overall survival in the univariate analysis. However, it failed to achieve the prognostic significance in multivariate prognostic analysis. High Ki-67 was significantly correlated with increased histologic grade. Of five histologic factors comprising of the histologic grade, correlation was found with necrosis, cell anaplasia, and especially with increased mitotic figures, but not with intracystic component, a factor correlated with p27 expression. This finding supports the primary importance of Ki-67 as a cell proliferation marker.

A correlation between p27 and Ki-67 expression has been reported in some malignant tumors (21, 22) but not in others. In our study, unpaired $t$ test and linear regression analysis failed to show association between p27 and Ki-67. p27 is highly expressed in cells arrested at the G0 and G1 phases, and Ki-67 is in cells at all cell cycle phases except the G0 phase (23). Therefore, the lack of correlation between these two molecules is partly explained by the fact that tumor cells arrested at the G1 phase express both p27 and Ki-67.

Selecting a subgroup of patients who may have a poor prognosis is clinically useful. In addition, a useful prognostic marker for this subgroup would permit the assignment of adjuvant therapy. p27 may be an important risk factor for both diseasefree and overall survivals of the patients who have MECA of the intraoral minor salivary gland. The prognostic importance of p27 expression should further be established in large-scale prospective studies.

Acknowledgments: We thank Motoo Yokoi, D.M.D., Department of Oral Surgery, Nagoya City University Hospital, Nagoya, for his expert advice. We also thank Ms. E. Tochizawa and Ms. C. Ando for their invaluable technical assistance.

\section{REFERENCES}

1. Waldron CA, el Mofty SK, Gnepp DR. Tumors of the intraoral minor salivary glands. A demographic and histologic study of 426 cases. Oral Surg Oral Med Oral Pathol 1988;66:323-33.

2. Melrose RJ, Abrams AM, Howell FV. Mucoepidermoid tumors of the intraoral minor salivary glands: a clinicopathologic study of 54 cases. J Oral Pathol 1973;2:314-25.

3. Olsen KD, Devine KD, Weiland LH. Mucoepidermoid carcinoma of the oral cavity. Otolaryngol Head Neck Surg 1981; 89:783-91.

4. Smitheringale A, Noyek AM, Chapnik JS, Blair RL, Freeman JL. Mucoepidermoid carcinoma of the palate. J Otolaryngol 1981;10:261-6.

5. Auclair PL, Goode RK, Ellis GL. Mucoepidermoid carcinoma of intraoral salivary glands. Evaluation and application of grading criteria in 143 cases. Cancer 1992;69:2021-30.

6. Yin HF, Okada N, Takagi M. Apoptosis and apoptotic-related factors in mucoepidermoid carcinoma of the oral minor salivary glands. Pathol Int 2000;50:603-9.

7. Toyoshima H, Hunter T. p27, a novel inhibitor of G1 cyclinCdk protein kinase activity, is related to p21. Cell 1994;78: 67-74.

8. Polyak K, Kato JY, Solomon MJ, Sherr CJ, Massague J, Roberts $\mathrm{JM}$, et al. $\mathrm{p} 27^{\mathrm{Kip} 1}$, a cyclin-Cdk inhibitor, links transforming 
growth factor-beta and contact inhibition to cell cycle arrest. Genes Dev 1994;8:9-22.

9. Polyak K, Lee MH, Erdjument BH, Koff A, Roberts JM, Tempst $\mathrm{P}$, et al. Cloning of $\mathrm{p} 27^{\mathrm{Kip} 1}$, a cyclin-dependent kinase inhibitor and a potential mediator of extracellular antimitogenic signals. Cell 1994;78:59-66.

10. Hengst L, Reed SI. Translational control of p $27^{\text {Kip1 }}$ accumulation during the cell cycle. Science 1996;271:1861-4.

11. Lloyd RV, Erickson LA, Jin L, Kulig E, Qian X, Cheville JC, et al. $227^{\mathrm{kip} 1}$ : a multifunctional cyclin-dependent kinase inhibitor with prognostic significance in human cancers. Am J Pathol 1999;154:313-23.

12. Mineta H, Miura K, Suzuki I, Takebayashi S, Amano H, Araki $\mathrm{K}$, et al. Low p27 expression correlates with poor prognosis for patients with oral tongue squamous cell carcinoma. Cancer 1999;85:1011-7.

13. Gerdes J, Schwab U, Lemke H, Stein H. Production of a mouse monoclonal antibody reactive with a human nuclear antigen associated with cell proliferation. Int J Cancer 1983; $31: 13-20$

14. Skalova A, Leivo I, Von Boguslawsky K, Saksela E. Cell proliferation correlates with prognosis in acinic cell carcinomas of salivary gland origin. Immunohistochemical study of 30 cases using the MIB 1 antibody in formalin-fixed paraffin sections. J Pathol 1994;173:13-21.

15. Nordgard S, Franzen G, Boysen M, Halvorsen TB. Ki-67 as a prognostic marker in adenoid cystic carcinoma assessed with the monoclonal antibody MIB1 in paraffin sections. Laryngoscope 1997;107:531-6.

16. Skalova A, Lehtonen H, von Boguslawsky K, Leivo I. Prognostic significance of cell proliferation in mucoepidermoid carcinomas of the salivary gland: clinicopathological study using MIB 1 antibody in paraffin sections. Hum Pathol 1994; 25:929-35.

17. Spiro RH, Thaler HT, Hicks WF, Kher UA, Huvous AH, Strong EW. The importance of clinical staging of minor salivary gland carcinoma. Am J Surg 1991;162:330-6.

18. Inagaki H, Nagasaka T, Otsuka T, Sugiura E, Nakashima N, Eimoto T. Association of SYT-SSX fusion types with proliferative activity and prognosis in synovial sarcoma. Mod Pathol 2000;13:482-8.

19. Pagano M, Tam SW, Theodoras AM, Beer-Romero P, Del Sal $\mathrm{G}$, Chau V, et al. Role of the ubiquitin-proteasome pathway in regulating abundance of the cyclin-dependent kinase inhibitor p27. Science 1995;269:682-5.

20. Fuse T, Tanikawa M, Nakanishi M, Ikeda K, Tada T, Inagaki $\mathrm{H}$, et al. $\mathrm{p} 27^{\mathrm{Kip} 1}$ expression by contact inhibition as a prognostic index of human glioma. J Neurochem 2000;74: 1393-9.

21. Sanchez BM, Saez AI, Martinez MJC, Sol Mateo M, Sanchez VL, Villuendas R, et al. Cyclin-dependent kinase inhibitor p2 $7^{\mathrm{KIP} 1}$ in lymphoid tissue: p27KIP1 expression is inversely proportional to the proliferative index. Am J Pathol 1997;151: 151-60.

22. Lloyd RV, Jin L, Qian X, Kulig E. Aberrant p27 $7^{\mathrm{kip} 1}$ expression in endocrine and other tumors. Am J Pathol 1997;150:401-7.

23. Gerdes J, Li L, Schlueter C, Duchrow M, Wohlengerg C, Gerlach C, et al. Immunobiochemical and molecular biologic characterization of the cell proliferation-associated nuclear antigen that is defined by monoclonal antibody Ki-67. Am J Pathol 1991;138:867-73. 